\title{
A long time ago, in a galaxy not so far away
}

Vast stellar nurseries, clouds that dwarf the Solar System and lurking swarms of black holes. Jeff Kanipe probes the unfolding mysteries at the heart of the Milky Way.

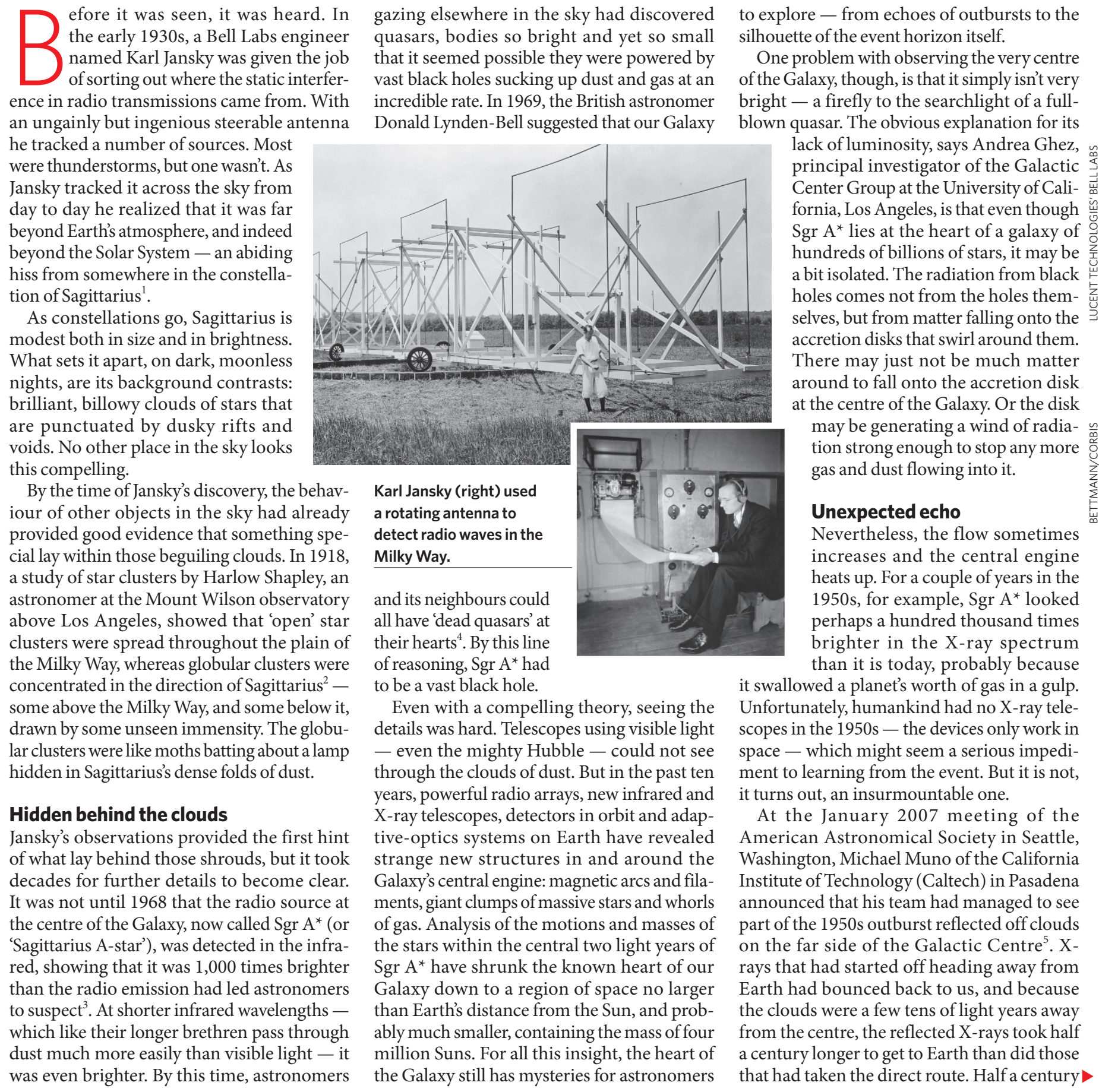


NEWS FEATURE

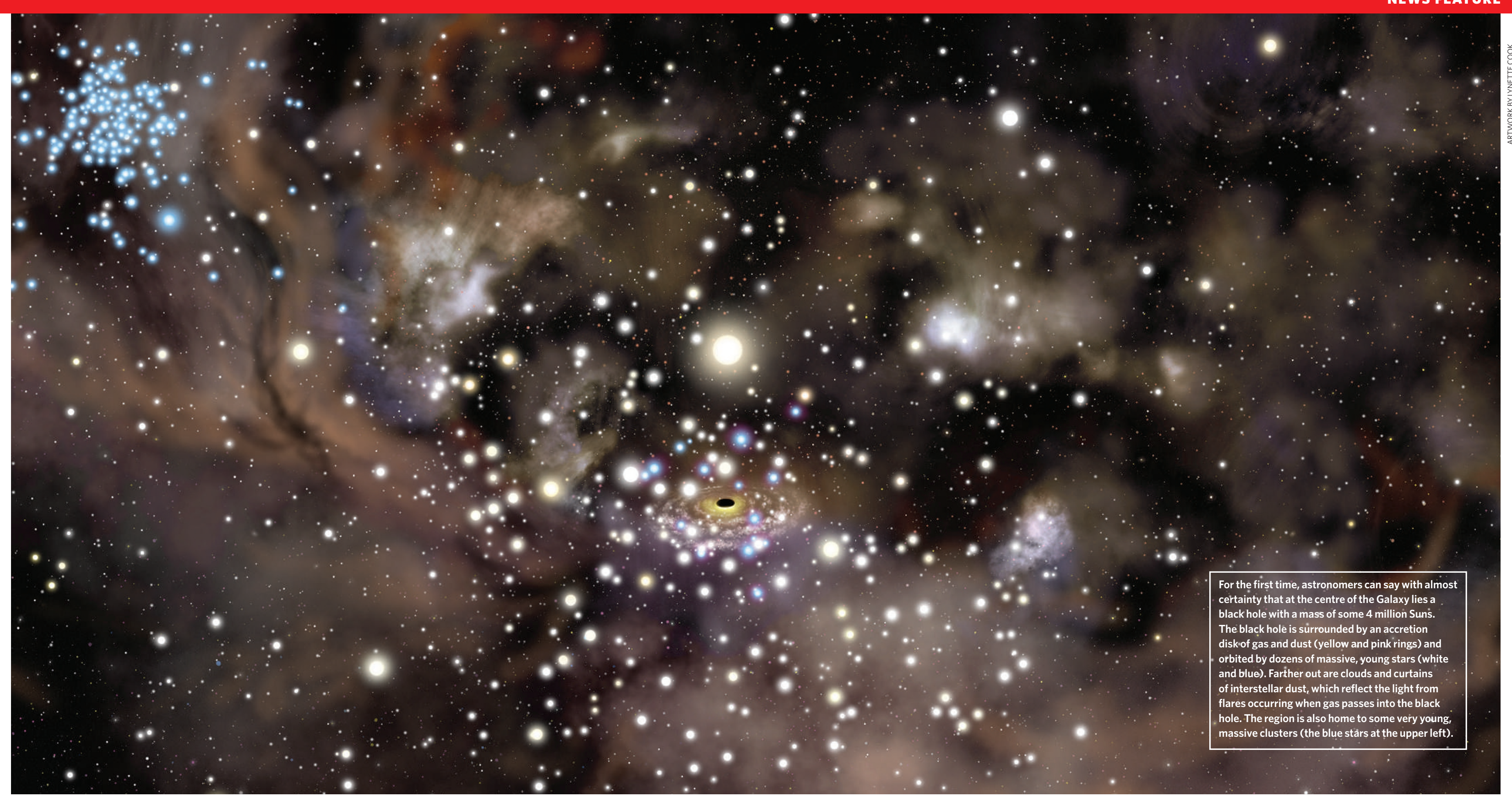

isn't much in terms of a journey across the
26,000 light years that separate Earth from the
events could imply that the disk of material $\begin{array}{lll}\text { Galactic Centre but it's enough to make the } & \text { swirling about the black hole is both meagre } & \text { something that weighs as much as four million } \\ \text { difference between astronomers stuck under- } & \text { and unstable, only occasionally dropping a } & \text { stars is sitting next door. }\end{array}$ neath Earth's X-ray-absorbing atmosphere and astronomers who, like Muno's team, can use the centre of the Galaxy. "This is the first $\mathrm{X}$ -

$\mathrm{X}$-ray echo that we have seen propagating through space after an even The observations allowed his team to say that the burstmusthave been 1,000 times brighter holes's inactivity suggests that the central few and 1,000 times longer than the contemporary to make stars. And the enormous gravitation Japanese Advanced Satellite for Cosmology to prohibit stars from forming even if the mate stars is sitting next doo obbet of matter into the black holes's maw. B and fomily of that $\mathrm{A}^{*} \mathrm{c}$ Sor $\mathrm{A}^{*}$ with a period of just over 15 years. At its closest, it comes within 17 light hours of the supermassive black hole ${ }^{6}$ - as close as the edge of our Solar System is to Earth. After 12 years of monitoring the motions of these stars using the infrared capabilities and adaptive optics of
the W. M. Keck Observatory on Mauna Kea, Hawaii, Ghezs Galactic Center Group, has could have occurred if the density of the
almost seen them make complete circuits of
gases in the centre of the Galaxy was much the centre and return to where they started: higher in the past. Higher density would We should see $\mathrm{S} 0-2$ close [its orbit] in 2010," she says. The orbits of the central stars of the Galaxy can be used to further refine the strain the distribution of mass in the neighbourhood. And their motions might also reveal something about how they got there in the first place.

There are two explanations for the that of the Sun, packed into a volume of
no greater than 3 light years, which is more
is compact than any cluster known. At the today, near the black hole. In principle, this there later on as a single massive custer. original cluster would have needed a mass
that was ten million times greater than

allow clumps in the clouds to collapse to form stars, even in the presence of a strong gravitational field

The alternative explanation is that the ditions of the central regio adverse conHowever, for this to work the core of the he first scenario

But although young stars may not be igrating into the central zone, very Gerists at the

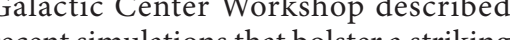
riction first made by Mark Morris of the University of California Los Angeles in 1993 . Morris postulated that the inner three light years of the Galaxy's centre 政 the remnants previous generations of bright young 作 the central and much larger black hole over 


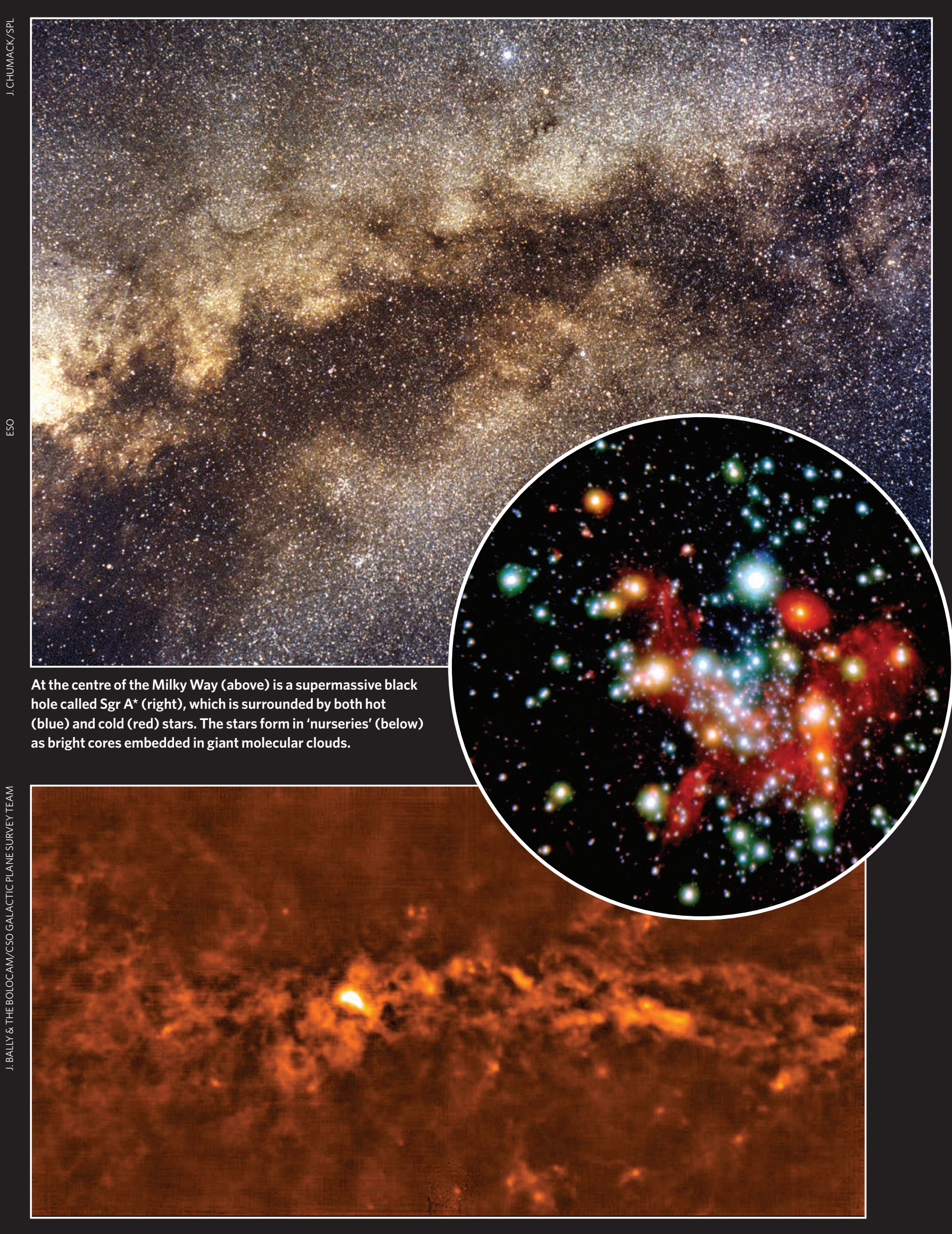

billions of years. The presence of a
close-knit cluster of dead stars is supported by Chandra’s discovery of four bright but variable X-ray sources - within 3 light years of Sgr
$\mathrm{A}^{*}($ ref. 7$)$. The sources variability i $\mathrm{A}^{*}($ ref. 7$)$. The sources' variability is
a characteristic of systems in which matter from a normal star is sucked neutron star Four fairly easily discerned X-ray sources of this type such a confined region, say astronomers, provide strong circumstantia evidence that tens of thousands of black holes and neutron stars have settled in and around Sgr A

Smouldering stars

To see some real clusters, we have years from Sor $\mathrm{A}^{*}$ lie the Arches an Quintuplet clusters - two of the most massive young clusters and unlike either the open or globular clusters seen elsewhere. The stars
in the Arches cluster are 50 times closer together than are those in the

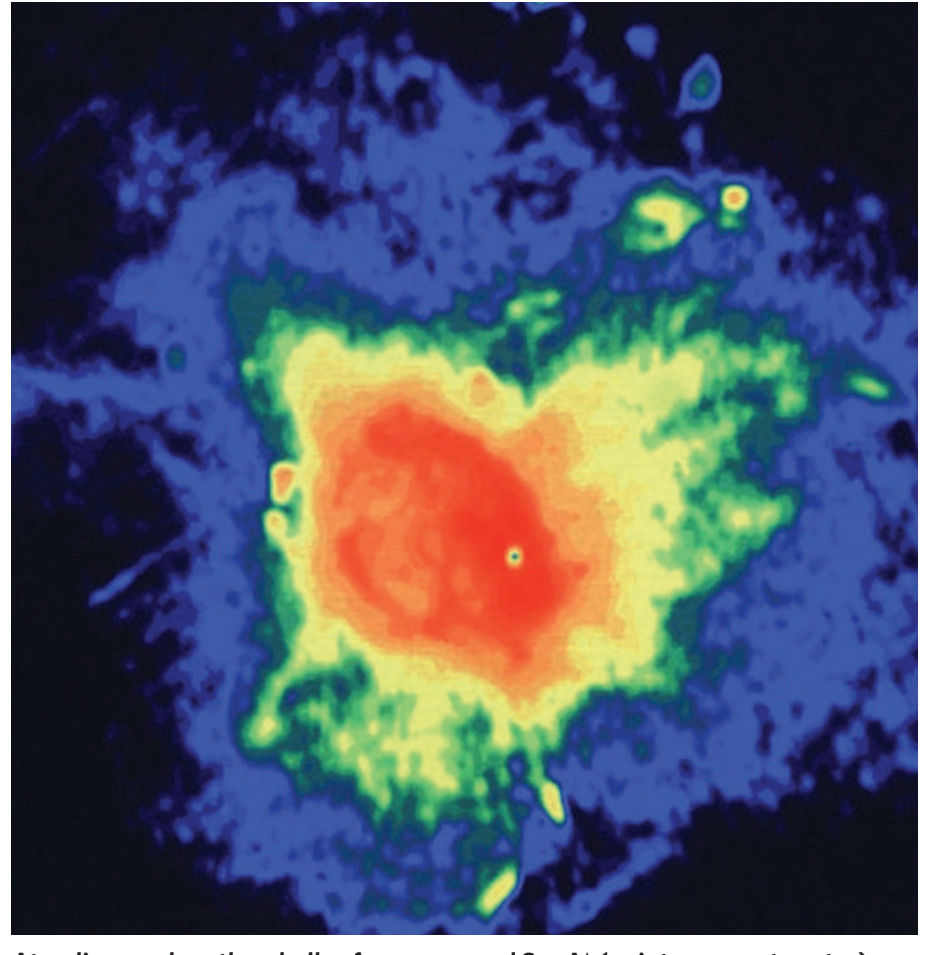

neighourhood of our Solar System. At the density seen in the Arches cluster, the space between the Sun and its nearest neighcluster the Quintuplet is a bit dispersed, but it has one of the biggest and potentially most volatile stars known, called the pistol star (so named for the pistol-shape nebula in which it lies)

The source of these mammoth sta clusters are giant molecular clouds - cool, dense complexes of dust and hydrogen gas
up to 130 light years in breadth and containup to 130 light years in breadth and contain-
ing the mass of between 10,000 and 500,000 ing the mass of between 10,000 and 500,000 Spitzer space telescope can sose on NASA's Spitzer space telsscope can see the parts the the infrared. Astronomers working at longer wavelengths can see even earlier stages of the star-birth process. New maps of the Galactic Centre made at the Caltech Submillimeter Observatory (CSO) on Mauna Kea, Hawail, reveal objects so early on in their developmen that they cant yet be called stars. "What we see are usually cores of clouds that have not necessarily begun to form stars yet, or are Mills, who is a member of CSO' Bolocan Galactic Plane Survey "With millimetre-wave data you get a more unbiased census of where star formation begins to occur. You see all of these nurseries, and whether or not they have a baby' in them yet"

A mosaic of these maps reveals a differen 604

The Galaxy's dark heart much like frosted glass blurs

an image," says Bower.

Bower and his peers

hope that new generation

of interferometers working

at millimetre and submilli-

metre wavelengths, which

black holes's event horizon

How would that look to

outside observers? Depend-

ing on its orich

tronomers think that the

elativistic effects of the black

feld would make the conal

horizon appear as a large

shadow or a silhouette cast

on a background of bright

plasma, in which the shadow

passes into the throat of the

black hole itself.

This image can be made with a network of milliters seen in Spitzer images are diminished; metre- and submillimetre-wavelength telethe cores of cooler clouds blossom with light, scopes distributed around he Earth, Bower massive protostars. These recions, which Others, including the largest the Atycist. form a ridge-like structure in images taken at Large Millimeter Array are under construcmillimetre wavelengths might one day form tion. Lashing them together into an ad-hoc a chain of clusters like those in the Arches or interferometer the size of Earth, though, is a Quintuplet, unless gravitational forces from daunting technological challenge. That, says the black hole disrupt their formation. Such Bower, "is part of the thrill of the chase". structures, says John Bally, the principal Such an observation would, of course, be a investigator of the survey, "are unique to the milestone - the first direct proof that an event Galactic Centre region."

The one structure that is absolutely unique,
though, is the supermassive black hole itself Given its apparent size and proximity to
Earth, says Geoffrey Bower of the University of California, Berkeley, it affords astronomers their best chance to image the black holes event horizon - the boundary beyond which no light can escape.

At the moment, the best observation of the black hole and its accretion disk ar hose made by a technique that links radro baseline interferometry At adistance of 26,000 light years an interferometer working a wavelengths with a baseline the size of a plan should be able to resolve details as small as the orbit of the Earth. Unfortunately, the radio waves from Sgr $\mathrm{A}$ " pass through intervening regions of highly ionized gas, which scatter it radio emissions. "These random distortion horizon, and therefore a black hole, exists. And observing 'hot spots' orbiting the black way to test the effects of relisity in stro gravitational field an endeavour that has so far yielded ambiguous results ${ }^{9}$. The Galactic Centre may no longer be the mystery it was in Shapley or ansky's day, but the better known it is, the more remarkable it looks - and it promises to become even more remarkable before toolong.

1. Jansky, K. Proc. Inst. Rad. Eng. 232, 1158-1163 (1935). Observatory No. 152 (1918).
Becklin, E.E. \& Neugebauer, G. Astrophys.J. 151, 145-161

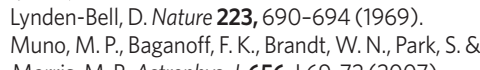
Morris, M. . R. Astrophys.J. 656, L69-72 (2007).
Ghez, A. M. etal. Astrophys. I. 586, L127-132 (2003). Muno, M. e.tal. Astrophys.J. 622 , L113-116 (2005). Broderick, A. . E. \& L Leeb, A. A. . . Phys. Conf. Ser. 54, 448-455
(2006) 